This manuscript is a draft (v. 1.0). Some material from this manuscript also appears in a related preprint, which is being split into two separate manuscripts due to length and topic separability: Sigurdardottir, H. M., Arnardottir, A., Halldorsdottir, E. T., Omarsdottir, H. R., \& Valgeirsdottir, A. S. (2019, July 16). Faces and words are both associated and dissociated: Evidence from visual problems in dyslexia. https://doi.org/10.31234/osf.io/n2kp6 An audio version of this previous preprint can be found at: https://notendur.hi.is/ heidasi/audio_papers/faces_and_words_v2/ Questions and comments are welcome and should be directed to the corresponding author.

\title{
Reading problems and their connection with visual search and attention: The search for a cause continues
}

\author{
Heida Maria Sigurdardottir ${ }^{1}$, Hilma Ros Omarsdottir ${ }^{2}$, \& Anna Sigridur \\ Valgeirsdottir ${ }^{2}$ \\ Icelandic Vision Lab, Department of Psychology, University of Iceland
}

1: Corresponding author (heidasi@hi.is)

2: These authors contributed equally and are listed alphabetically

\begin{abstract}
Attention has been hypothesized to act as a sequential gating mechanism for the orderly processing of letters in words. These same visuo-attentional processes are assumed to partake in some but not all visual search tasks. In the current study, 60 adults with varying degrees of reading abilities, ranging from expert readers to severely impaired dyslexic readers, completed an attentionally demanding visual conjunction search task thought to heavily rely on the dorsal visual stream. A visual feature search task served as an internal control. According to the dorsal view of dyslexia, reading problems should go hand in hand with specific problems in visual conjunction search - particularly elevated conjunction search slopes (time per search item) which would be interpreted as a problem with visual attention. Results showed that reading problems were associated with slower visual search, especially conjunction search. However, problems with reading were not associated with increased conjunction search slopes but instead with increased conjunction search intercepts, traditionally not interpreted as reflecting attentional processes. Our data are hard to reconcile with hypothesized problems in dyslexia with the serial moving of an attentional spotlight across a visual scene or a page of text.
\end{abstract}

Keywords: dyslexia, reading, visual attention, visual search, dorsal stream

Reading problems fall along a continuum where individuals tend to maintain their position over time (Shaywitz, Escobar, Shaywitz, Fletcher \& Makuch, 1992). People on the lowest part of this spectrum might get diagnosed with developmental dyslexia, a common learning disorder that primarily manifests as persistent failure to acquire efficient reading abilities (Démonet, Taylor, \& Chaix, 2004; Shaywitz \& Shaywitz, 2005; Shaywitz et al., 2003). Dyslexia is not generally thought of as a visual problem. According to what we will refer to as the dorsal view, some reading problems could nonetheless stem from a selective visual attentional disorder (reviewed elsewhere in e.g. Facoetti, Franceschini, \& Gori, 2019; Stein, 2014; Valdois, Bosse, \& Tainturier, 2004; Vidyasagar \& Pammer, 2010). The dorsal view has been gaining traction but is still met with healthy skepticism.

The dorsal stream plays a major role in representing space and guiding actions - including "covert actions" such as the orienting of visual attention - and is therefore often referred to as the 'where' or 'how' stream (see e.g. Brooks, Sigurdardottir, \& Sheinberg, 2014; Colby \& Goldberg, 1999; Milner \&
Goodale, 2006; Ungerleider \& Haxby, 1994; Ungerleider \& Mishkin, 1982). Subregions of the dorsal stream are structurally and functionally connected to ventral stream regions that partake in visual word and object recognition (Kay \& Yeatman, 2017; Vogel, Miezin, Petersen, \& Schlaggar, 2012; White, Palmer, Boynton, \& Yeatman, 2019) and which are functionally abnormal in dyslexic readers (Richlan et al., 2011; Sigurdardottir et al., 2015). Top-down activation stemming from dorsal stream regions seemingly serves to amplify the stimulus selectivity for words and other objects already computed by high-level ventral stream regions (Kay \& Yeatman, 2017).

These same dorsal regions and their nonhuman primate homologues have been the focus of extensive research on the neuroanatomical substrates of visual search as they guide the eyes and attention to important objects or locations (Brooks et al., 2014; Baluch \& Itti, 2011; Bisley \& Goldberg, 2010; Colby \& Goldberg, 1999; Fecteau \& Munoz, 2006; Sigurdardottir, 2017). Vidyasagar and Pammer (2010) argued that visuo-attentional mechanisms controlled by the dorsal stream support both serial visual search and 
reading; just as serial visual search requires sweeping a spotlight of attention across a scene, attention is swept across the letters in a word during reading (see also e.g. Vidyasagar, 2013; Vidyasagar \& Pammer, 1999). Attentional priority signals bias the activity of neurons within ventral stream areas toward the object of attention even when multiple objects or visual features are present within a neuron's receptive field (Brooks et al., 2014). Rapid movements of attention across a word could therefore act as a sequential gating mechanism for visual information, ensuring that the ventral stream processes the letters in order and thus facilitating the recognition of the word (Vidyasagar \& Pammer, 2010). Any disruptions in such visuoattentional processes could lead to problems with both serial visual search and reading.

Not all visual search is however traditionally thought to require the serial moving of an attentional spotlight. So-called conjunction search is often assumed to require serial shifts of attention (but see c.f. Townsend, 1990) around the display where visual features in an attended location are bound into a perceptual whole. Stimuli in feature search are however often assumed to be processed in parallel without shifts of visual attention, making the search minimally attention demanding (Arguin, Joanette, \& Cavanagh, 1993; Treisman \& Gelade, 1980; for alternative interpretations, see e.g. Eckstein, 2011; Kristjánsson, 2015).

In feature search, a searched-for target stimulus is defined by a unique visual feature not shared by distractors, such as when a single white line (target) is shown amongst several black lines (distractors). Search for a salient target with a unique feature can be very efficient with a near-zero slope of reaction times with respect to the number of items in the search display (set size); the target appears to pop out and the time to find it becomes largely independent of the number of distractors (Scialfa \& Joffe, 1998; Treisman \& Gelade, 1980; Wang, Kristjansson, \& Nakayama, 2005; Wolfe \& Gray, 2007). In conjunction search, the target shares visual features with distractors and thus can only be defined by a unique combination of features, such as when the target is a white horizontal line amongst white vertical and black horizontal distractors. Conjunction search tends to be comparatively inefficient, with reaction time slopes markedly greater than zero; search time thus linearly increases with set size (Scialfa \& Joffe, 1998; Treisman \& Gelade, 1980; Wang et al., 2005; Wolfe \& Gray, 2007). Compared to feature search, conjunction search is generally thought to rely more on the dorsal stream (Arguin et al., 1993; Ashbridge, Walsh, \& Cowey, 1997; Corbetta, Shulman, Miezin, \& Petersen, 1995; Ellison, Rushworth, \& Walsh,
2003; Robertson, Treisman, Friedman-Hill, \& Grabowecky, 1997) although this could have more to do with differences in search efficiency rather than differential reliance on feature binding (Donner et al., 2002; Nobre, Coull, Walsh, \& Frith, 2003).

As conjunction search and reading have been proposed to both involve the sequential moving of an attentional spotlight from item to item, we briefly summarize all studies that we could find on the performance of dyslexic readers on classic (i.e. noncued single target among distractors) visual search tasks with non-alphabetical stimuli (Buchholz \& McKone, 2004; Casco \& Prunetti, 1996; de BoerSchellekens \& Vroomen, 2012; Facoetti, Paganoni, \& Lorusso, 2000; Hayduk, Bruck, \& Cavanagh, 1996; Iles, Walsh, \& Richardson, 2000; Jones, Branigan, \& Kelly, 2008; Nguyen et al., 2021; Roach \& Hogben, 2004; Sireteanu, Goebel, Goertz, \& Wandert, 2006; Sireteanu et al., 2008; Vidyasagar \& Pammer, 1999; Wright, Conlon, \& Dyck, 2012).

There is little evidence for any deficiencies in efficient feature search tasks in dyslexia (with a notable exception in Hayduk et al., 1996). Two studies (Facoetti et al., 2000; Jones et al., 2008) reported problems with feature search tasks, but typical readers in both showed worse performance with increased set size so the search was likely not efficient. There is stronger evidence supporting that dyslexic readers have difficulties with inefficient search such as conjunction search, although samples in several studies have been quite small. This has generally been interpreted as a problem with visual attention consistent with the dorsal view.

It is nonetheless not as clear whether these problems are exacerbated by increased numbers of distracting items in the search display, i.e., whether there are search slope differences between dyslexic and typical readers. Search slopes that measure response time changes with increased set size have traditionally been used to assess the speed of search and the involvement of attention (Kristjánsson, 2015; Wolfe, 2016). This is a crucial measure for any claims of a visual attentional deficit in reading problems. Conjunction search tasks are generally thought to require serial attentional selections of search items, where visual attention moves (overtly or covertly) to a new search item, the item is recognized as a target or dismissed as a distractor, in which case attention is disengaged and finally moves to a different item. Any general problems with serial attentional selection, such as the proposed sluggish attentional shifting in dyslexia (Hari \& Renwall, 2001), should manifest as elevated conjunction search slopes. 
To foreshadow our results, people with reading problems tended to be slower at conjunction search, and this general speed of conjunction search explained unique variability in reading problems not explained by performance in feature search or measures of ADHD (e.g. inattention, impulsiveness). However, elevated conjunction search slopes were not demonstrably associated with reading problems, and conjunction search slopes were not predictive of lexical decision slopes (time per letter in a word or pseudoword), i.e. word length effects. These results do not easily fit with the view that reading difficulties are caused by problems with sequential attentional gating of visual information (e.g. letters, search items). Implications for the dorsal view are discussed.

\section{Method}

\section{Participants}

60 people participated, thereof 48 women and 12 men. Mean age was 25 years (range 19 to 51). All participants were undergraduate students or had recently graduated. They were required to have Icelandic as their native language and to report normal or correctedto-normal vision. The participants were selected by convenience and were volunteers; people with reading problems were likely overrepresented. The stopping rule for data collection was to test a minimum of 60 volunteers but to stop collecting data if 80 people had been tested before a certain time point. 24 participants were deemed to be dyslexic (reported previous formal diagnosis of dyslexia or screened positive for dyslexia on the Adult Reading History Questionnaire with an ARHQ score of 0.43 or higher) and 36 were judged to be typical readers. Mean age of both groups was 25 years, and gender ratios were similar (dyslexic readers: $20.8 \%$ males; typical readers: $19.4 \%$ males). Six participants were selected in a lottery to receive a gift certificate of 10.000 ISK.

\section{Procedure}

The study (protocol 14-027) was approved by the Icelandic National Bioethics Committee and reported to the Icelandic Data Protection Authority. Participants gave informed written consent. They were tested in a sound-attenuated chamber. First the participants filled out a questionnaire regarding former diagnoses of dyslexia and other disorders. They then completed three questionnaires: The Adult Reading History Questionnaire (ARHQ), designed to assess a history of reading problems indicative of dyslexia, and Behavioral Evaluation Questionnaire for Adults I and II, intended to assess symptoms of ADHD. The three questionnaires were computerized. Next, participants were placed in a chinrest to control for the viewing distance from the screen, which was approximately $57 \mathrm{~cm}$. They then partook in a face matching task measuring global form face matching and feature-based face matching; data from this task are analyzed in detail elsewhere (see Sigurdardottir et al., 2019). Thereafter came a visual search task measuring feature and conjunction search, after which the effect of word length on lexical decision time was measured. The chinrest was then removed, and participants finished the experiment by completing the Icelandic word form reading test (IS-FORM) and the Icelandic pseudoword form reading test (IS-PSEUDO). In cases where pre-recorded instructions were not used (see below), participants were given a choice of reading by themselves or having instructions read out loud to them. The same applied for the consent form. A 17-inch monitor (resolution $1024 \times 768$ ) with a refresh rate of 85 $\mathrm{Hz}$ was used for all tasks performed on a Dell OptiPlex 760 computer. Computerized tasks were run using PsychoPy (Peirce, 2007).

Adult Reading History Questionnaire. ARHQ, initially developed by Lefly and Pennington (Lefly \& Pennington, 2000), evaluates the reading history of adults. The Icelandic translation has been psychometrically tested in a large diverse sample of adults in Iceland where it was verified as a reliable screening tool for dyslexia (Bjornsdottir et al., 2014). Validity has been demonstrated by comparing adults with a diagnosis of specific reading disorder to typical adult readers (Bjornsdottir et al., 2014). ARHQ consists of 23 questions that are responded to on a five-point Likert-scale ranging from 0 to 4 . The total score is divided by the maximum score that gives a score ranging from 0 to 1 , with higher scores indicating more reading difficulties. A suggested cut-off point for dyslexia screening is a score of 0.43 or higher (Bjornsdottir et al., 2014). As recommended by Bjornsdottir et al. (2014), question number 15 in the Icelandic version is excluded in the analysis.

Behavioral Evaluation Questionnaires. The Behavioral Evaluation Questionnaires are self-report rating scales for adults regarding their current and former ADHD symptoms as defined by DSM-IV. Validity has been established in a sample of relatives of children and adolescents who were diagnosed with ADHD (Magnússon et al., 2006). These lists have been confirmed as reliable screening tools for ADHD symptoms (Magnússon et al., 2006). Each list consists of 18 questions on a 4-point Likert scale, resulting in a total score from 0 to 54 on each list, where higher scores imply more ADHD-related symptoms. Questionnaire I evaluates behavior in the last six months and questionnaire II evaluates behavior from 5 to 12 years of age. Score points for all questions are summed for 
each list separately to produce two total scores, one for current ADHD symptoms (ADHD-I) and the other for symptoms of ADHD during childhood (ADHD-II). Higher scores indicate increased ADHD symptoms.

Visual Search. Visual search was measured using short simple lines (approximately $1^{\circ}$ ). The lines were oriented 45 degrees to the left or right and were either black or white. Participants had to find a target line characterized by a unique combination of brightness (randomly chosen per trial) and orientation (counterbalanced for each set size) and thus differed from all the other distractor lines. A target was always present. When found, participants were to specify if the target was in the upper or lower half of the monitor using the up and down arrow keys, respectively. Set size of each trial was a minimum of 10 lines and a maximum of 60 lines. Set size could vary in increments of five (10 lines, 15 lines, 20 lines etc.). The locations of the target and distractors were selected randomly on each trial from 64 possible jittered locations on an invisible $8 \times 8$ grid with the most distant locations approximately $11^{\circ}$ from screen center (figure 1). Inter-trial interval was 200 ms.

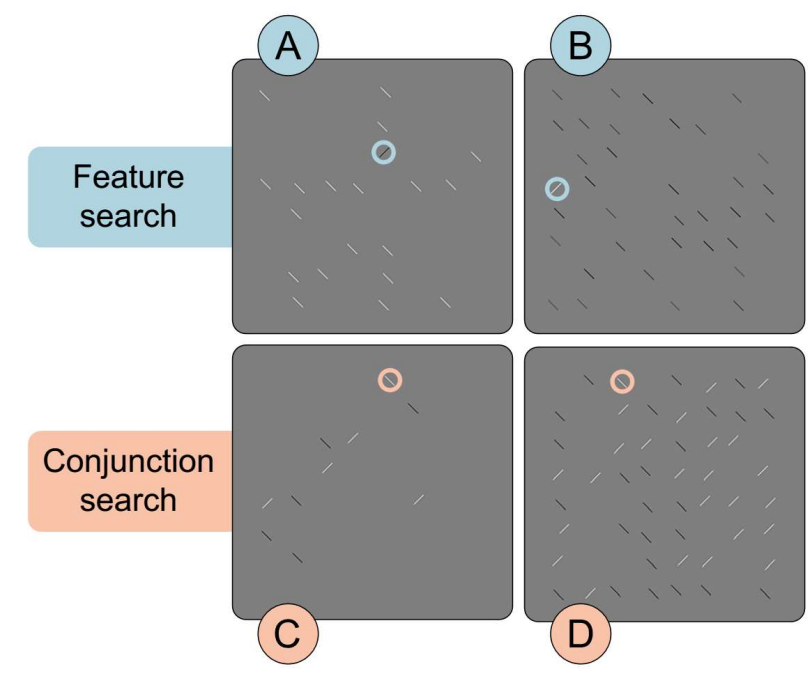

Figure 1. Example stimuli used for visual search. Top panel: Visual feature search with a set size of A) 20 stimuli and B) 35 stimuli, where the target has a distinctive brightness and orientation. Bottom panel: Visual conjunction search with a set size of C) 10 stimuli and D) 50 stimuli, where the target is defined by a unique combination of brightness and orientation. Participants indicated whether the target (always present) was found on the top (as shown here in A-D) or bottom half of the screen. The circle (not shown to participants) marks the target.

Participants listened to pre-recorded instructions for practice trials. The practice trials consisted of 12 trials which included intermixed feature and conjunction search trials with a variation of set sizes. During the practice trials, when the participant had given an answer regarding the target's position, distractors disappeared, and a horizontal line appeared across the middle of the monitor to indicate the decision boundary, i.e. where the upper and lower halves were divided. The target line and decision boundary line stayed onscreen for two seconds after a response was given.

On completion of the practice trials, participants listened to pre-recorded instructions preparing them for the experimental blocks. On experimental trials, all lines disappeared as soon as participants responded. The target thus did not stay onscreen, and no decision boundary line was shown in the middle of the monitor. Measures of feature and conjunction search were obtained in separate blocks of 66 trials each (6 of each set size). Blocks one and three measured feature search (132 trials) and blocks two and four measured conjunction search (132 trials). In feature search, the target had both a distinctive orientation and brightness different from all distractors (figure 1, upper panels). In conjunction search, the target had a specific combination of brightness and orientation not shared by distractors (figure 1, lower panels).

Lexical Decision. A lexical decision task was included to measure the word length effect, often defined as the relationship between naming time or lexical decision time and the number of letters in a word (for a review, see Barton, Hanif, Bjornstrom \& Hills, 2014). The word length effect is reduced as children develop reading skills - in other words, adding a letter minimally increases response times (Barton et al., 2014; Zoccolotti et al., 2005). An elevated word length effect persists in dyslexic readers (Juphard, Carbonnel, \& Valdois, 2004; Martens \& de Jong, 2006; Ziegler, Perry, Ma-Wyatt, Ladner, \& Schulte-Korne, 2003; Zoccolotti et al., 1999), consistent with a letter-by-letter reading strategy. A greatly increased word length effect is also a characteristic of reading deficits acquired through damage of ventral and dorsal brain regions (Barton et al, 2014; Behrmann, Nelson, \& Sekuler, 1998; Reinhart, Schaadt, Adams, Leonhardt, \& Kerkhoff, 2013), and which are associated with visual recognition or attentional problems. The word length effect was estimated to see whether it could be accounted for by dyslexic readers' potential visual problems, here more specifically assessed by elevated visual conjunction search slopes.

Words were selected from the Icelandic Frequency Dictionary (Pind, Magnússon, \& Briem, 1991). Words chosen for the test were Icelandic words with four, five, and six letters. Each word length category (four, five, or six letters) included 10 high frequency words and 10 low frequency words. Mean word frequency was not significantly different for the three word length categories (one-way ANOVA, F(2, 57) = 
$0.210, p=0.811)$. The distribution of word frequency was also not significantly different across word length categories (independent-samples Kruskal-Wallis test, $H(2)=3.815, p=0.148$ ). All words were concrete nouns with two syllables. Pseudowords were created by rearrangement. For example, the Icelandic words "ibúð" (e. apartment) and "magi" (e. stomach) would be rendered into two pseudowords, "ibgi" and "maúð". Ten pseudowords were created from the low frequency category words and the same was done for the high frequency category words. The test contains 20 real words and 20 pseudowords for each word length category, which adds up to 60 real words and 60 pseudowords in total. Stimuli were shown in a randomized order that was kept the same for all participants.

Participants listened to prerecorded instructions. The test included 120 trials separated by two breaks. On each trial, a fixation cross was displayed on the screen center for $500 \mathrm{~ms}$, after which it was replaced by a word or a pseudoword (Arial font, black letters, letter height approximately $1^{\circ}$ ). Participants were instructed to indicate as quickly but as accurately as possible with a key press whether the letter string was a word or a pseudoword. Response keys ' $\mathrm{G}$ ' and ' $\mathrm{H}$ ', marked with red stickers, were used to distinguish between words and pseudowords; keyboard mapping was counterbalanced. Participants received visual feedback for incorrect answers, where the incorrectly answered (pseudo)word appeared on the monitor for $500 \mathrm{~ms}$ in red color.

IS-FORM and IS-PSEUDO Reading Tests. ISFORM and IS-PSEUDO reading tests $(2015 ; 2017)$ measure (pseudo)words read per minute and percentage of correctly read (pseudo)words. IS-FORM includes two lists of 128 words each. One contains common Icelandic word forms and the other uncommon word forms (Icelandic is an inflected language so the same word can take many forms). IS-PSEUDO contains one list of 128 pseudowords.

The lists were presented one at a time. A page containing the list was placed in front of the participants with the backside up. Participants were told to start reading immediately after the experimenter had counted to three and turned the page around. Participants were instructed to read as fast as they possibly could while making as few errors as possible. The lists were presented in this order: common word forms, uncommon word forms, and pseudowords.

\section{Data Analysis and Exclusion/Inclusion}

Visual search trials and lexical decision trials with wrong answers were filtered out before reaction times were analyzed. Reaction times \pm 3 standard deviations away from a participant's mean were then excluded. An alpha level of 0.05 was used for statistical tests, which were all two-sided. Degrees of freedom in t-tests were adjusted in cases of a significant Levene's test for equality of variances. All participants were included in the analysis of the visual search tasks. Three participants were excluded from the analysis of the lexical decision task as they misunderstood the instructions $(0 \%, 6 \%$ and $7 \%$ correct answers, respectively). Recording of the reading of two participants failed for the IS-FORM common list, one participant had missing values for IS-FORM uncommon, and one for IS-PSEUDO. Linear regression was used to impute reading speed and accuracy for a missing list from the reading speed and accuracy of the two other lists before calculating each person's average reading speed (words per minute) and accuracy (percent of correctly read words) across the three reading lists.

\section{Results}

\section{Reading Measures}

Adult Reading History Questionnaire (ARHQ) scores, reading speed, and reading accuracy were our main reading measures. Dyslexic readers tended to read more poorly than typical readers according to all measures (table 1).

\begin{tabular}{l|lllllc}
\hline \multicolumn{1}{c}{ Dyslexic } & \multicolumn{2}{c}{ Typical } & & \\
& \multicolumn{1}{c}{$M$} & $S D$ & \multicolumn{1}{c}{$M$} & $S D$ & $\mathrm{p}$ & $\mathrm{d}$ \\
\hline ARHQ & 0.6 & 0.12 & 0.3 & 0.07 & NA & 3.099 \\
Read. speed & 61 & 14.8 & 82 & 15.6 & $<0.001$ & 1.440 \\
Read. acc. & 90.6 & 6.77 & 96.5 & 2.38 & $<0.001$ & 1.163 \\
ADHD-I & 16.6 & 7.73 & 8.8 & 5.29 & $<0.001$ & 1.185 \\
ADHD-II & 22.5 & 13.7 & 9.9 & 7.37 & $<0.001$ & 1.182 \\
\hline
\end{tabular}

Table 1. Group differences in reading and ADHD symptoms. ADHD-I measures current ADHD symptoms and ADHD-II measures childhood ADHD symptoms according to the Behavioral Evaluation Questionnaires for Adults I and II. M: mean; SD: standard deviation; p: probability; $d$ : Cohen's d. Read. speed: Reading speed in words per minute. Read. acc.: Reading accuracy percent correctly read words. No $p$-values are reported for ARHQ group differences as ARHQ scores directly affected group membership.

\section{ADHD Measures}

Two participants, both dyslexic, reported a previous diagnosis of attention deficit hyperactivity disorder (ADHD), consistent with the well-known comorbidity of the two disorders (Germanò, Gagliano, \& Curatolo, 2010). Dyslexic readers tended to report greater current 
as well as childhood symptoms of ADHD (table 1). Below, we estimate to which degree ADHD symptoms (e.g. inattention, impulsiveness) can account for patterns in the data.
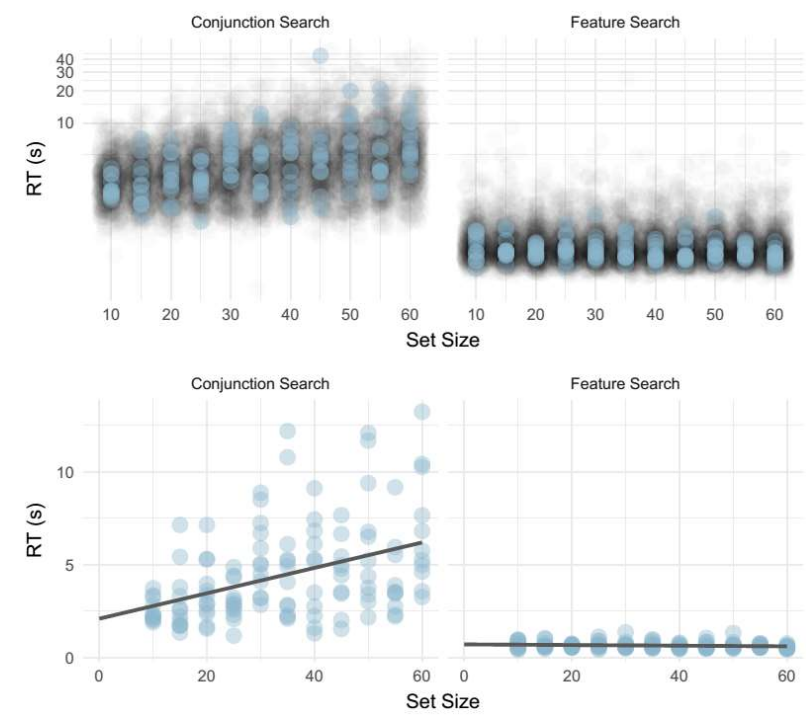

Figure 2. Response times of correct trials in conjunction search and feature search. Upper panels: Response times (RTs) for all correct trials from all participants (black markers, jittered to minimize overlap) as well as from a single example participant (blue markers). Logarithmic scale is used to visualize both short and long RTs. Lower panels: Response times for all correct trials of the same example participant after extreme RT values $( \pm 3$ standard deviations away from the mean) have been removed. RT scale is linear. Linear fits for RTs relative to set size in conjunction search (left) and feature search (right) are shown as gray lines. Intercepts and slopes were estimated in this way for each individual participant.

\section{Visual Search}

As expected, response times tended to increase with set size for conjunction search but not feature search (figure 2, upper panels). For each participant, response times were regressed on set size separately for conjunction and feature searches (figure 2, lower panels). The intercept (predicted response times for an infinitely small set size) and slope (predicted increase in response times for each element added to the search display) were then used in further analysis.

Overall group differences and correlations. Dyslexic readers tended to have higher search intercepts than typical readers in conjunction search (figure 3; dyslexic readers $M=3075 \mathrm{~ms}, S D=920$; typical readers $M=2561 \mathrm{~ms}, S D=569 ; t(34.784)=$ $2.445, p=0.020, d=0.672$ ). The conjunction search intercept was also significantly correlated with $A R H Q$ $(r(58)=0.338, p=0.008)$ and reading speed $(r(58)=-$ $0.329, p=0.010)$ but not reading accuracy $(r(58)=$ $0.209, p=0.108)$. The general speed of conjunction search was therefore associated with reading problems, as slow conjunction searchers were more likely to be dyslexic, reported a greater history of reading problems, and read slower.

The group difference in feature search intercepts did not reach significance (dyslexic readers $M=862 \mathrm{~ms}, S D=240$; typical readers $M=762 \mathrm{~ms}, S D$ $=139 ; t(33.394)=1.847, p=0.074, d=0.510$; figure 3 ). The feature search intercept was however significantly correlated with reading speed $(r(58)=-0.347, p=0.007)$ but not with ARHQ $(r(58)=0.240, p=0.065)$ or reading accuracy $(r(58)=0.083, p=0.527)$. Slow feature search was therefore associated with slow reading.

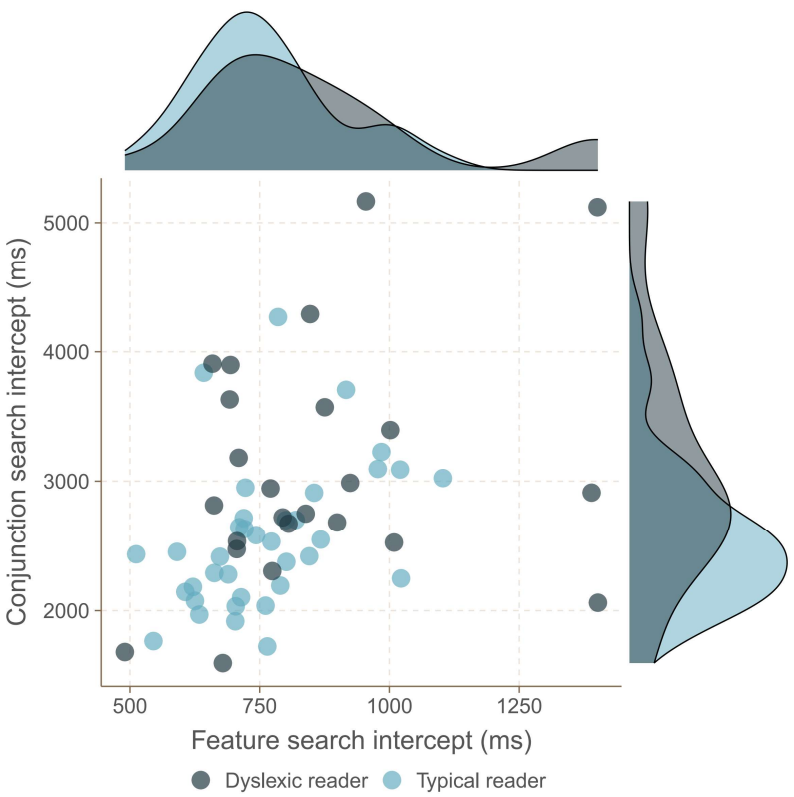

Figure 3. Estimated intercepts of dyslexic (black) and typical (blue) readers in conjunction and feature search. Each dot corresponds to one participant. Marginal plots show density estimates for the two groups.

No significant group differences were found in either search task for search slopes. Conjunction search slopes for dyslexic and typical readers were comparable and high (dyslexic readers $M=46 \mathrm{~ms}, S D$ $=25$; typical readers $M=43 \mathrm{~ms}, S D=21 ; t(58)=0.563$, $p=0.575, d=0.146)$ and feature search slopes for both groups were flat (dyslexic readers $M=-1 \mathrm{~ms}, S D=4$; typical readers $M=-1 \mathrm{~ms}, S D=2 ; t(31.615)=0.651, p$ $=0.520, d=0.181)$. Search slopes were not significantly correlated with any of the three reading measures (all absolute $r \mathrm{~s}<0.191$, all $p s>0.144)$. As group comparisons for conjunction search slopes were particularly theoretically important, we followed up with a Bayesian independent samples t-test which indicated moderate evidence (Lee \& Wagenmakers, 2014) in favor of the null hypothesis of no differences in slopes $\left(\mathrm{BF}_{01}=3.281\right)$. 
Specific effects: Regression models. We wanted to estimate whether problems with visual search were independent of or driven by symptoms of ADHD (e.g. inattention, impulsiveness), whether problems with conjunction search were associated with reading problems over and above more general problems with visual search, and finally whether these problems were especially expressed as elevated conjunction search slopes. We performed a hierarchical logistic regression with group membership as the dependent variable and three hierarchical linear regression models with $A R H Q$, reading speed, and reading accuracy as dependent variables. ADHD-I scores, ADHD-II scores, and ADHD diagnosis (0: not diagnosed; 1: diagnosed) were entered at stage 1 of each model. Feature search intercept and slope were entered at stage 2 , conjunction search intercept at stage 3 , and finally conjunction search slope at stage 4 .

\begin{tabular}{l|lccc}
\hline \multicolumn{1}{c}{} & \multicolumn{2}{c}{ Group } & \multicolumn{3}{c}{ ARHQ } \\
& -7.712 & 0.002 & 0.052 & 0.650 \\
\hline Constant & 0.122 & 0.097 & 0.008 & 0.047 \\
ADHD-I & 0.065 & 0.130 & 0.003 & 0.247 \\
ADHD-II & 17.902 & 0.999 & 0.130 & 0.311 \\
ADHD diagnosis & 3.795 & 0.139 & 0.033 & 0.816 \\
Feature intercept & 135.590 & 0.421 & -4.775 & 0.572 \\
Feature slope & 0.672 & 0.224 & 0.052 & 0.109 \\
Conjunction intercept & -0.699 & 0.966 & 0.834 & 0.377 \\
Conjunction slope & Reading & speed & Reading & accuracy \\
& \multicolumn{1}{c}{$b$} & $p$ & $b$ & $p$ \\
\hline Constant & 114.367 & $<0.001$ & 0.975 & $<0.001$ \\
ADHD-I & -0.420 & 0.356 & 0.001 & 0.345 \\
ADHD-II & -0.170 & 0.545 & -0.002 & 0.011 \\
ADHD diagnosis & -1.809 & 0.901 & 0.032 & 0.456 \\
Feature intercept & -12.740 & 0.426 & 0.065 & 0.176 \\
Feature slope & 1287.466 & 0.184 & 0.912 & 0.749 \\
Conjunction intercept & -6.780 & 0.068 & -0.021 & 0.062 \\
Conjunction slope & -62.007 & 0.564 & -0.267 & 0.404 \\
\hline
\end{tabular}

Table 2. Summary of regression models with group membership (logistic regression, $\chi^{2}(7)=30.072, p<0.001$, $R^{2}$ Nagelkerke $=0.533$ ), ARHQ (linear regression, $F(7,52)=4.897$, $p<0.001, R^{2}=0.397, R^{2}$ adjusted $=0.316$ ), reading speed (linear regression, $F(7,52)=2.611, p=0.022, R^{2}=0.260, R^{2}$ adjusted $=$ 0.160 ) and reading accuracy (linear regression, $F(7,52)=$ 2.328, $p=0.038, R^{2}=0.239, R^{2}$ adjusted $=0.136$ ) as dependent variables, and measures of ADHD and visual search as independent variables. Unstandardized regression coefficients (b) and p-values are bold for significant independent predictors (blue when $b$ is positive and red when $b$ is negative). Regression coefficients for search slopes and intercepts are in seconds.

ADHD measures were predictive of group membership and reading measures (excluding reading speed) as was evident from stage 1 models (group membership model, $\chi^{2}(3)=23.739, p<0.001$, $R^{2}$ Nagelkerke $=0.442 ; \mathrm{ARHQ}$ model $(F(3,56)=9.301, p<$
$0.001, R^{2}=0.333, R^{2}$ adjusted $=0.297$; reading accuracy model $\left(F(3,56)=3.268, p=0.028, R^{2}=0.149, R^{2}\right.$ adjusted $=0.103$; reading speed model $(F(3,56)=2.383, p=$ $0.079, R^{2}=0.113, R^{2}$ adjusted $=0.066$ ).

Adding feature search parameters at stage 2 did not significantly improve any models (model change for group membership: $\chi^{2}(2)=4.766, p=0.092$, $R^{2}$ Nagelkerke change $=0.069$; for $\mathrm{ARHQ}: F(2,54)=0.710$, $p=0.496, R^{2}$ change $=0.017$; for reading speed: $F(2,54)=2.868, p=0.065, R^{2}$ change $=0.085$; for reading accuracy: $F(2,54)=0.493, p=0.613, R^{2}$ change $=0.015$ ). Feature search performance thus did not explain a significant portion of the variance not already explained by measures of ADHD.

Adding the conjunction search intercept at stage 3 did not improve the predictive ability of the logistic regression model for group membership (model change: $\chi^{2}(1)=1.565, p=0.211, R^{2}$ Nagelkerke change $=$ $0.022)$ nor did it significantly improve the prediction of ARHQ scores (model change: $F(1,53)=3.331, p=$ $0.074, R^{2}$ change $=0.038$ ). It did however significantly improve the prediction of both reading speed $(F(1,53)=$ 4.048, $p=0.049, R^{2}$ change $\left.=0.057\right)$ and reading accuracy $\left(F(1,53)=4.394, p=0.041, R^{2}\right.$ change $=$ $0.064)$. The general speed of conjunction search thus appeared to capture some of the variance in reading not explained by measures of ADHD or feature search.

Finally, adding the conjunction slope at stage 4 did not improve any models (model change for group membership: $\chi^{2}(1)=0.002, p=0.966, R^{2}$ Nagelkerke change < 0.001; for ARHQ: $F(1,52)=0.794, p=0.377$, $R^{2}$ change $=0.009$; for reading speed: $F(1,52)=0.338$, $p=0.564, R^{2}$ change $=0.005$; for reading accuracy: $F(1,52)=0.707, p=0.404, R^{2}$ change $=0.010$. Stage 4 models are summarized in table 2 . Note that while all stage 4 regression models were overall significant and explained a considerate proportion of the variance in their respective dependent variables, few individual factors were significant, and no model had any visual search measures as significant independent predictors. Any one factor thus mostly seemed to be explaining the same part of the variance in reading problems as one or more other factors included in the models.

Visual search accuracy. Our main goal was to estimate response time intercepts and slopes for the two visual search tasks. While we found some group differences in search speed, this could hypothetically be due to a group difference in an emphasis on speed vs. accuracy. We therefore additionally looked at visual search accuracy. No reliable group differences were found in overall accuracy for either feature search (dyslexic readers $M=98.4 \%, S D=1.53$; typical readers $M=98.6 \%, S D=1.12 ; t(58)=0.738, p=0.463, d=$ 0.188 ) or conjunction search (dyslexic readers $M=$ 
$96.1 \%, S D=3.96$; typical readers $M=97.1 \%, S D=$ 1.84; $t(29.715)=1.227, p=0.229, d=0.344)$.

An exploratory analysis (hence, no statistical tests) of group differences in accuracy by target location, initially done to look for signs of a left-sided minineglect in dyslexic readers attributed to a right parietal lobe hypofunction (Hari, Renvall, \& Tanskanen, 2001; no obvious hints of such neglect were found), revealed an interesting pattern depicted in figure 4 . In our search tasks, the location of a target (always present) had to be reported as being on the top or bottom half of the screen. Naturally, this decision was particularly challenging for targets close to the invisible horizontal midline of the monitor so both groups tended to make more errors when reporting the location of such targets. However, errors rose markedly for some dyslexic readers when targets were close to this decision boundary, particularly for conjunction search but also somewhat in feature search. Future studies are needed to test by confirmatory analysis whether this pattern reflects a true effect.

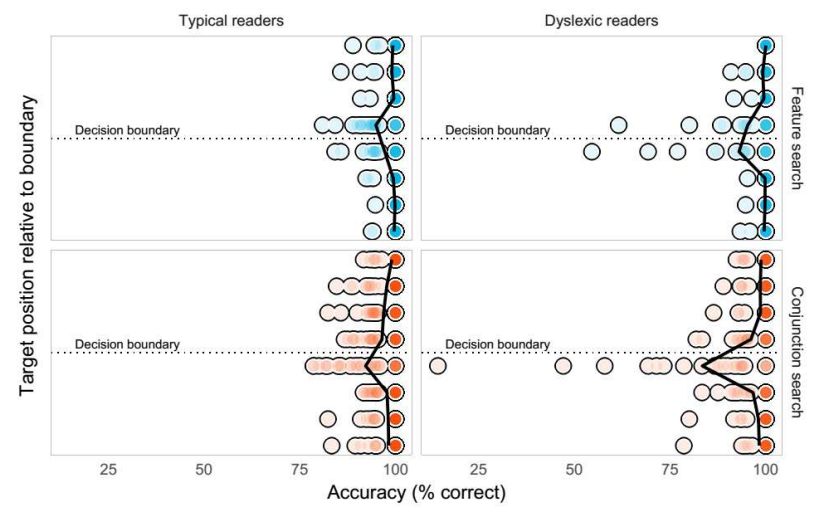

Figure 4. Accuracy of feature search (blue, top panels) and conjunction search (red, bottom panels) for typical and dyslexic readers by the location of a target relative to a decision boundary. The target could appear in eight possible vertical locations, thereof four above an invisible horizontal midline (decision boundary) of the screen and four below. A participant's accuracy is represented by eight dots, one for each vertical target location. Dots with stronger colors indicate multiple participants. The solid black line marks the mean accuracy.

\section{Lexical Decision}

In the lexical decision task, a response time slope was estimated for each individual participant in the same manner as described for visual search, except that "set size" in the lexical decision task was defined as the number of letters in each word or pseudoword. The slope corresponds to the predicted increase in response times for each letter added to a word or pseudoword, i.e. the word length effect. Dyslexic readers tended to have a larger word length effect compared to typical readers (dyslexic readers $M=35 \mathrm{~ms}, S D=47$; typical readers $M=7 \mathrm{~ms}, S D=22 ; t(26.966)=2.660, p=0.013$, $d=0.775$ ). As no reliable group differences were found in visual conjunction search slopes, then unsurprisingly there was no significant correlation between the word length effect and conjunction search slopes $(r(55)=-$ $0.195, p=0.146$ ).

Although estimating the slope (word length effect) was our primary reason for running the lexical decision task, we do note that estimated lexical decision intercepts (i.e. predicted response times for an infinitely short word/pseudoword) are correlated with both feature search intercepts $(r(55)=0.427, p<0.001)$ and conjunction search intercepts $(r(55)=0.532, p<0.001)$. Those who are slow at visual search therefore tend to be slow at deciding whether letters form a valid word or a pseudoword, but while each added letter tends to increase lexical decision times more for dyslexic readers than typical readers (i.e. greater slope for dyslexic readers), each added conjunction search item does not increase response time to a greater degree for dyslexic readers than it does for typical readers (i.e. comparable slopes), and there is no detectable relationship between the slopes from these two tasks (see also Supplementary Information for additional exploratory analysis).

\section{Discussion}

We asked our participants to perform two visual search tasks where they had to find a unique target and report whether it was on the top or bottom of the screen. One was feature search, often thought to be minimally attention demanding, where the target popped out because it had a unique brightness and orientation. The other was conjunction search where the target was defined by a combination of features. Unlike feature search, conjunction search and reading have been proposed to both involve the serial moving of an attentional spotlight from item to item. According to this line of thinking, one would expect reading problems to be specifically associated with problems with visual conjunction search. More specifically, as the high slopes in conjunction search have traditionally been interpreted as reflecting the moving of an attentional spotlight, one would expect that a problem in such an attentional process would be reflected in heightened search slopes for conjunction search.

This is however not exactly what we found. As expected, dyslexic readers had problems with conjunction search. These were however reflected in heightened search intercepts while there was no detectable group difference in search slopes. Intercept differences are not traditionally thought to reflect attentional differences as long as the slopes are 
constant and are instead often interpreted as a difference in some other process that either precedes or follows the actual visual search (Kristjánsson, 2015) where "the actual visual search" could involve factors such as attentional shifting/focusing, attentional engagement, and episodic retrieval (Ramgir \& Lamy, 2020). If these assumptions are valid, it is of crucial importance for any claims of visual attentional problems in dyslexia to look for evidence for slope changes, which were not found. Wolfe (2016) suggests that slopes provide an index of the amount of guidance and of the rate of processing of selected items, while changes to the non-search portion of a task will generally lead to intercept changes. According to this line of thinking, dyslexic and typical readers show no consistent differences in guidance or rate of processing. We do note that slopes have been suggested to be an ambiguous measure of attentional involvement, and intercept differences could be interpreted as attentional differences according to some models of visual attention (Kristjánsson, 2015; see also Buetti, Cronin, Madison, Wang, \& Lleras, 2016; Eckstein, 1998).

Another pattern of results was not as expected. There were hints that reading problems went together with difficulties not just in conjunction search but also in feature search, although likely to a lesser degree. Previous results generally agree that poor reading is not associated with any problems in efficient feature search tasks. One possible clue comes from the type of errors made. Unlike many feature search tasks, our participants were always required to locate the target as being above or below an invisible decision boundary at the midline of the monitor. The errors of some dyslexic readers markedly increased close to this decision boundary in both search tasks. So, one possible - but speculative - way of interpreting our data is that dyslexic readers have problems with precisely locating visual stimuli. This would fit with the general idea of a dorsal stream deficit in dyslexia. It should more generally be noted that while it is often assumed that the dorsal stream does not partake in feature search, inactivation of dorsal stream areas important for overt and covert visual attention (Sigurdardottir, 2017) increases target detection times even in feature search tasks (Wardak, Olivier, \& Duhamel, 2004).

Vidyasagar \& Pammer (2010) argue that letters in words need to be serially recognized, that this requires sequential attentional gating, and that a deficiency in such a visuo-spatial mechanism could lead to problems with both serial visual search and reading in dyslexia. An increased word length effect could therefore theoretically be associated with the slope of conjunction search; the search slope is traditionally thought to measure time per search item just like the word length effect measures time per letter, and both could reflect the serial moving of attention from one visual stimulus to another. However, while dyslexic readers as a group showed signs of an increased word length effect, indicative of letter-by-letter reading, this was not consistently associated with visual search.

The dual-route theory of reading describes two reading routes, one lexical and another nonlexical, which have been respectively described as a dictionary lookup procedure and a letter-to-sound rule procedure (Coltheart, Curtis, Atkins, \& Haller, 1993). A diminished word length effect in efficient readers is in accordance with increased reliance on lexical processing where letters are processed in parallel (Barton et al, 2014) while letter-by-letter reading in dyslexic readers could be based on deficient nonlexical processing where graphemes are serially decoded into phonemes (Martens \& de Jong, 2006). This possibility is in accordance with the current finding, as well as with previous results (e.g. Coltheart et al., 2001), that word length effects are driven by pseudowords which - by definition - cannot be successfully processed by a lexical route. An increased word length effect in some dyslexic readers could reflect phonological problems independent of any visuoattentional problems.

To conclude, reading problems were associated with slower visual search, especially attentionally demanding conjunction search. However, our data do not support the idea that reading problems are specifically associated with problems in the serial moving of an attentional spotlight across a visual scene or a page of text.

\section{Author Note}

This work was supported by The Icelandic Research Fund (Grant No. 174013-051) and the University of Iceland Research Fund. Data can be made available to other researchers upon request provided that the National Bioethics Committee of Iceland grants them permission for such access and provided that such access adheres to all Icelandic laws regarding data privacy and protection.

We wish to thank Andrey Chetverikov for his assistance with the coding of the visual search tasks, and Alexandra Arnardottir and Eydis Thuridur Halldorsdottir for collaboration with running participants. We also want to thank Ómar Ingi Jóhannesson for his assistance, and Árni Kristjánsson for helpful discussions. Finally, we want to thank Sabrina Hansmann-Roth for her idea of making audio versions of the Icelandic Vision Lab's papers. Data from this manuscript have previously been presented at conferences and in theses. 
This manuscript is a draft (v. 1.0). Some material from this manuscript also appears in a related preprint, which is being split into two separate manuscripts due to length and topic separability: Sigurdardottir, H. M., Arnardottir, A., Halldorsdottir, E. T., Omarsdottir, H. R., \& Valgeirsdottir, A. S. (2019, July 16). Faces and words are both associated and dissociated: Evidence from visual problems in dyslexia. https://doi.org/10.31234/osf.io/n2kp6 An audio version of this previous preprint can be found at: https://notendur.hi.is/ heidasi/audio_papers/faces_and_words_v2/ Questions and comments are welcome and should be directed to the corresponding author.

\section{References}

Arguin, M., Joanette, Y., \& Cavanagh, P. (1993). Visual search for feature and conjunction targets with an attention deficit. Journal of Cognitive Neuroscience, 5(4), 436-452.

Ashbridge, E., Walsh, V., \& Cowey, A. (1997). Temporal aspects of visual search studied by transcranial magnetic stimulation. Neuropsychologia, 35(8), $1121-1131$.

Baluch, F., \& Itti, L. (2011). Mechanisms of top-down attention. Trends in Neurosciences, 34(4), 210224.

Barton, J. J., Hanif, H. M., Eklinder Björnström, L., \& Hills, C. (2014). The word-length effect in reading: A review. Cognitive Neuropsychology, 31(5-6), 378-412.

Behrmann, M., Nelson, J., \& Sekuler, E. B. (1998). Visual complexity in letter-by-letter reading: Pure alexia is not pure. Neuropsychologia, 36(11), 11151132.

Bisley, J. W., \& Goldberg, M. E. (2010). Attention, intention, and priority in the parietal lobe. Annual Review of Neuroscience, 33, 1-21.

Bjornsdottir, G., Halldorsson, J. G., Steinberg, S., Hansdottir, I., Kristjansson, K., Stefansson, H., \& Stefansson, K. (2014). The Adult Reading History Questionnaire (ARHQ) in Icelandic: Psychometric properties and factor structure. Journal of Learning Disabilities, 47(6), 532-542.

Brooks, D., Sigurdardottir, H. M., \& Sheinberg, D. L. (2014). The neurophysiology of attention and object recognition in visual scenes in. In $\mathrm{K}$. Kveraga \& M. Bar (Eds.), Scene Vision (pp. 85104). Cambridge, MA: MIT Press.

Buchholz, J., \& McKone, E. (2004). Adults with dyslexia show deficits on spatial frequency doubling and visual attention tasks. Dyslexia, 10(1), 24-43.

Buetti, S., Cronin, D. A., Madison, A. M., Wang, Z., \& Lleras, A. (2016). Towards a better understanding of parallel visual processing in human vision: Evidence for exhaustive analysis of visual information. Journal of Experimental Psychology: General, 145(6), 672-707.

Casco, C., \& Prunetti, E. (1996). Visual search of good and poor readers: Effects with targets having single and combined features. Perceptual and Motor Skills, 82(3_suppl), 1155-1167.

Colby, C. L., \& Goldberg, M. E. (1999). Space and attention in parietal cortex. Annual Review of Neuroscience, 22(1), 319-349.

Coltheart, M., Curtis, B., Atkins, P., \& Haller, M. (1993). Models of reading aloud: Dual-route and parallel-distributed-processing approaches Psychological Review, 100(4), 589-608

Coltheart, M., Rastle, K., Perry, C., Langdon, R., \& Ziegler, J. (2001). DRC: a dual route cascaded model of visual word recognition and reading aloud. Psychological Review, 108(1), 204-256.

Corbetta, M., Shulman, G. L., Miezin, F. M., \& Petersen, S. E. (1995). Superior parietal cortex activation during spatial attention shifts and visual feature conjunction. Science, 270(5237), 802-805. de Boer-Schellekens, L., \& Vroomen, J. (2012). Sound can improve visual search in developmental dyslexia. Experimental Brain Research, 216(2), 243-248.

Démonet, J.-F., Taylor, M. J., \& Chaix, Y. (2004). Developmental dyslexia. The Lancet, 363(9419), 1451-1460.

Donner, T. H., Kettermann, A., Diesch, E., Ostendorf, F., Villringer, A., \& Brandt, S. A. (2002). Visual feature and conjunction searches of equal difficulty engage only partially overlapping frontoparietal networks. Neurolmage, 15(1), 1625.

Eckstein, M. P. (1998). The lower visual search efficiency for conjunctions is due to noise and not serial attentional processing. Psychological Science, 9(2), 111-118.

Eckstein, M. P. (2011). Visual search: A retrospective. Journal of Vision, 11(5), 14-14.

Ellison, A., Rushworth, M., \& Walsh, V. (2003). The parietal cortex in visual search: a visuomotor hypothesis. In Supplements to Clinical Neurophysiology (Vol. 56, pp. 321-330): Elsevier.

Facoetti, A., Franceschini, S., \& Gori, S. (2019). Role of visual attention in developmental dyslexia. In $\mathrm{L}$. Verhoeven, C. Perfetti, \& K. Pugh (Eds.), Developmental Dyslexia across Languages and Writing Systems, pp. 307-326. Cambridge: Cambridge University Press.

Facoetti, A., Paganoni, P., \& Lorusso, M. L. (2000). The spatial distribution of visual attention in developmental dyslexia. Experimental Brain Research, 132(4), 531-538.

Fecteau, J. H., \& Munoz, D. P. (2006). Salience, relevance, and firing: A priority map for target selection. Trends in Cognitive Sciences, 10(8), 382-390.

Germanò, E., Gagliano, A., \& Curatolo, P. (2010). Comorbidity of ADHD and dyslexia. Developmental Neuropsychology, 35(5), 475493.

Hari, R., \& Renvall, H. (2001). Impaired processing of rapid stimulus sequences in dyslexia. Trends in Cognitive Sciences, 5(12), 525-532.

Hari, R., Renvall, H., \& Tanskanen, T. (2001). Left minineglect in dyslexic adults. Brain, 124(7), 1373-1380

Hayduk, S., Bruck, M., \& Cavanagh, P. (1996). Low level visual processing skills of adults and children with dyslexia. Cognitive Neuropsychology, 13(7), 975-1016.

Ho, J., Tumkaya, T., Aryal, S., Choi, H., \& Claridge-Chang, A. (2019). Moving beyond $P$ values: data analysis with estimation graphics. Nature Methods, 1.

Iles, J., Walsh, V., \& Richardson, A. (2000). Visual search performance in dyslexia. Dyslexia, 6(3), 163177

Jones, M. W., Branigan, H. P., \& Kelly, M. L. (2008). Visual deficits in developmental dyslexia: relationships between non-linguistic visual tasks and their contribution to components of reading. Dyslexia, 14(2), 95-115. 
Juphard, A., Carbonnel, S., \& Valdois, S. (2004). Length effect in reading and lexical decision: Evidence from skilled readers and a developmental dyslexic participant. Brain and Cognition, 55(2), 332-340.

Kay, K. N., \& Yeatman, J. D. (2017). Bottom-up and topdown computations in word-and face-selective cortex. Elife, 6, e22341.

Kristjánsson, A. (2015). Reconsidering visual search. iPerception, 6(6), 2041669515614670.

Lee, M. D., \& Wagenmakers, E. J. (2014). Bayesian cognitive modeling: A practical course. Cambridge University Press.

Lefly, D. L., \& Pennington, B. F. (2000). Reliability and validity of the adult reading history questionnaire. Journal of Learning Disabilities, 33(3), 286-296

Magnússon, P., Smári, J., Sigurðardóttir, D., Baldursson, G., Sigmundsson, J., Kristjánsson, K., . . Guð̌mundsson, Ó. Ó. (2006). Validity of selfreport and informant rating scales of adult ADHD symptoms in comparison with a semistructured diagnostic interview. Journal of Attention Disorders, 9(3), 494-503.

Martens, V. E., \& de Jong, P. F. (2006). The effect of word length on lexical decision in dyslexic and normal reading children. Brain and Language, 98(2), 140-149.

Milner, D., \& Goodale, M. (2006). The Visual Brain in Action. Oxford, UK: Oxford University Press.

Reinhart, S., Schaadt, A. K., Adams, M., Leonhardt, E., \& Kerkhoff, G. (2013). The frequency and significance of the word length effect in neglect dyslexia. Neuropsychologia, 51(7), 1273-1278.

Nguyen, B. N., Kolbe, S. C., Verghese, A., Nearchou, C. McKendrick, A. M., Egan, G. F., \& Vidyasagar, T. R. (2021). Visual search efficiency and functional visual cortical size in children with and without dyslexia. Neuropsychologia, 107819.

Nobre, A., Coull, J., Walsh, V., \& Frith, C. (2003). Brain activations during visual search: contributions of search efficiency versus feature binding. Neurolmage, 18(1), 91-103.

Peirce, J. W. (2007). PsychoPy—psychophysics software in Python. Journal of Neuroscience Methods, 162(1-2), 8-13.

Pind, J., Magnússon, F., \& Briem, S. (1991). Íslensk orđtíđnibók. Reykjavik, Iceland: Orðabók Háskólans.

Ramgir, A., \& Lamy, D. (2020, September 4). Top-down, bottom-up and selection-history-based control of attention: A failed trichotomy? The case of Priming of pop-out $(P o P)$. PsyArXiv. https://doi.org/10.31234/osf.io/4zy6q

Richlan, F., Kronbichler, M., \& Wimmer, H. (2011). Metaanalyzing brain dysfunctions in dyslexic children and adults. Neurolmage, 56(3), 1735-1742.

Roach, N. W., \& Hogben, J. H. (2004). Attentional modulation of visual processing in adult dyslexia: a spatial-cuing deficit. Psychological Science, 15(10), 650-654

Robertson, L., Treisman, A., Friedman-Hill, S., \& Grabowecky, M. (1997). The interaction of spatial and object pathways: Evidence from Balint's syndrome. Journal of Cognitive Neuroscience, 9(3), 295-317.
Scialfa, C. T., \& Joffe, K. M. (1998). Response times and eye movements in feature and conjunction search as a function of target eccentricity. Perception \& Psychophysics, 60(6), 1067-1082.

Shaywitz, S. E., Escobar, M. D., Shaywitz, B. A., Fletcher, J. M. \& Makuch, R. (1992). Evidence that dyslexia may represent the lower tail of a normal distribution of reading ability. The New England Journal of Medicine, 326(3), 145-150.

Shaywitz, S. E., \& Shaywitz, B. A. (2005). Dyslexia (specific reading disability). Biological Psychiatry, 57(11), 1301-1309.

Sigurdardottir, H. M. (2017). Lateral Intraparietal Region (LIP). In J. Vonk \& T. Shackelford (Eds.), Encyclopedia of Animal Cognition and Behavior. New York: Springer.

Sigurdardottir, H. M., Arnardottir, A., Halldorsdottir, E. T., Omarsdottir, H. R., \& Valgeirsdottir, A. S. (2019). Faces and words are both associated and dissociated: Evidence from visual problems in dyslexia. PsyArXiv. https://doi.org/10.31234/osf.io/n2kp6

Sigurdardottir, H. M., Ívarsson, E., Kristinsdóttir, K., \& Kristjánsson, Á. (2015). Impaired recognition of faces and objects in dyslexia: Evidence for ventral stream dysfunction? Neuropsychology, 29(5), 739-750

Sireteanu, R., Goebel, C., Goertz, R., \& Wandert, T. (2006). Do children with developmental dyslexia show a selective visual attention deficit? Strabismus, 14(2), 85-93.

Sireteanu, R., Goebel, C., Goertz, R., Werner, I. Nalewajko, M., \& Thiel, A. (2008). Impaired serial visual search in children with developmental dyslexia. Annals of the New York Academy of Sciences, 1145(1), 199-211.

Stein, J. (2014). Dyslexia: The role of vision and visual attention. Current Developmental Disorders Reports, 1(4), 267-280.

Treisman, A. M., \& Gelade, G. (1980). A featureintegration theory of attention. Cognitive Psychology, 12(1), 97-136.

Townsend, J. T. (1990). Serial vs. parallel processing: Sometimes they look like Tweedledum and Tweedledee but they can (and should) be distinguished. Psychological Science, 1(1), 4654.

Ungerleider, L. G., \& Haxby, J. V. (1994). 'What'and 'where' in the human brain. Current Opinion in Neurobiology, 4(2), 157-165

Ungerleider, L. G., \& Mishkin, M. (1982). Two cortical visual systems. In D. J. G. Ingle, M. A.; Mansfield, R. J. W. (Ed.), Analysis of visual behavior (pp. 549-586). Cambridge, MA: MIT Press.

Valdois, S., Bosse, M. L., \& Tainturier, M. J. (2004). The cognitive deficits responsible for developmental dyslexia: Review of evidence for a selective visual attentional disorder. Dyslexia, 10(4), 339363.

Vidyasagar, T. R. (2013). Reading into neuronal oscillations in the visual system: implications for developmental dyslexia. Frontiers in Human Neuroscience, 7, 811. 
Vidyasagar, T. R., \& Pammer, K. (1999). Impaired visual search in dyslexia relates to the role of the magnocellular pathway in attention. NeuroReport, 10(6), 1283-1287.

Vidyasagar, T. R., \& Pammer, K. (2010). Dyslexia: a deficit in visuo-spatial attention, not in phonological processing. Trends in Cognitive Sciences, 14(2), 57-63.

Vogel, A. C., Miezin, F. M., Petersen, S. E., \& Schlaggar, B. L. (2012). The putative visual word form area is functionally connected to the dorsal attention network. Cerebral Cortex, 22(3), 537-549.

Wang, D., Kristjansson, A., \& Nakayama, K. (2005).

Efficient visual search without top-down or bottom-up guidance. Perception \& Psychophysics, 67(2), 239-253.

Wardak, C., Olivier, E., \& Duhamel, J.-R. (2004). A deficit in covert attention after parietal cortex inactivation in the monkey. Neuron, 42(3), 501 508.

White, A. L., Palmer, J., Boynton, G. M., \& Yeatman, J. D. (2019). Parallel spatial channels converge at a bottleneck in anterior word-selective cortex. Proceedings of the National Academy of Sciences, 116(20), 10087-10096.

Wolfe, J. M. (2016). Visual search revived: the slopes are not that slippery: a reply to Kristjansson (2015). i-Perception, 7(3), 2041669516643244

Wolfe, J. M., \& Gray, W. (2007). Guided search 4.0. In Gray W. (Ed.), Integrated Models of Cognitive Systems (pp. 99-119). New York: Oxford..

Wright, C. M., Conlon, E. G., \& Dyck, M. (2012). Visual search deficits are independent of magnocellular deficits in dyslexia. Annals of Dyslexia, 62(1), 53-69.

Zoccolotti, P., De Luca, M., Di Pace, E., Judica, A., Orlandi, M., \& Spinelli, D. (1999). Markers of developmental surface dyslexia in a language (Italian) with high grapheme-phoneme correspondence. Applied Psycholinguistics, 20(2), 191-216 


\section{Supplementary Information}

\section{Lexical Decision}

Dyslexic readers tended to be slower (figure s1) and less accurate (figure s2) than typical readers in the lexical decision task. This was true for both high and low frequency words, but the group difference in mean response times was especially apparent for pseudowords. Both groups were highly accurate for high frequency words, but dyslexic readers made more errors for low frequency words (indicating that they were pseudowords) and pseudowords (indicating that they were real words).

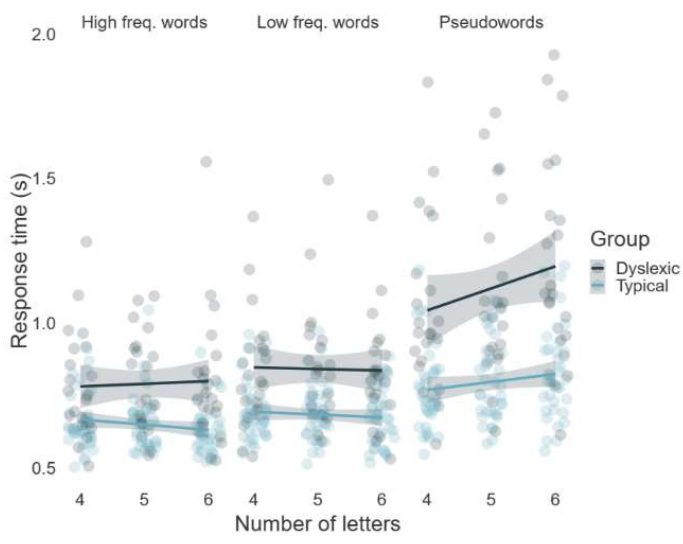

Figure s1. Mean response times (RTs) for lexical decision by the number of letters in a (pseudo)word, frequency of word (high, low, none), and group membership. Only correct trials were included, and trials over three standard deviations from the RT mean of each participant were additionally thrown out. Each dot shows a participant's mean RT for a particular combination of letter number and word frequency. Dots are jittered to minimize overlap. Lines show linear fits for each group. Shaded areas indicate 95\% confidence boundaries.

Word length effects were driven by pseudowords. Previous research has similarly reported a length effect on naming latencies for pseudowords and an absence of a length effect for real words (Coltheart, Rastle, Perry,
Langdon, \& Ziegler, 2001), and more generally a negative relationship is found between word frequency and word length effects (Barton et al., 2014). In the current data, response times increased, and accuracy decreased for longer pseudowords, and this pattern was exacerbated for dyslexic readers compared to typical readers. Neither low nor high frequency real words in either group showed apparent response time increases or accuracy decreases with increased word length.

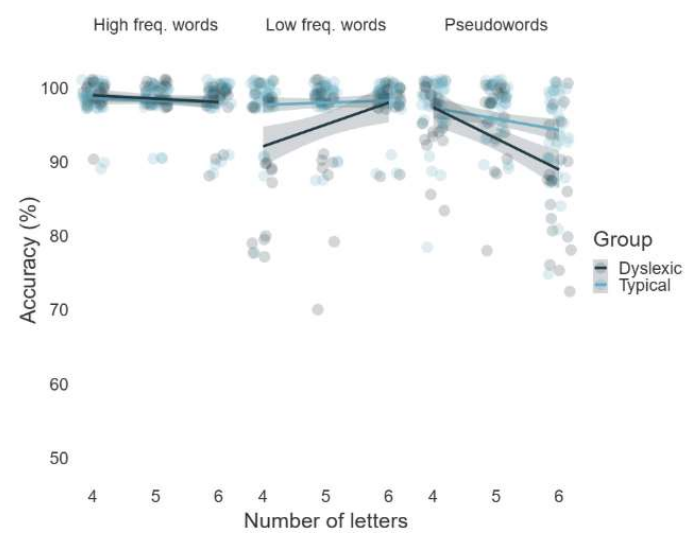

Figure s2. Accuracy (percent correct) for lexical decision by the number of letters in a (pseudo)word, frequency of word (high, low, none), and group membership. All trials were included. Each dot shows a participant's mean RT for a particular combination of letter number and word frequency. Dots are jittered to minimize overlap. Lines show linear fits for each group. Shaded areas indicate 95\% confidence boundaries.

\section{Supplementary References}

Barton, J. J., Hanif, H. M., Eklinder Björnström, L., \& Hills, C. (2014). The word-length effect in reading: A review. Cognitive Neuropsychology, 31(5-6), 378-412.

Coltheart, M., Rastle, K., Perry, C., Langdon, R., \& Ziegler, J. (2001). DRC: a dual route cascaded model of visual word recognition and reading aloud. Psychological Review, 108(1), 204-256. 